ORIGINAL ARTICLE

\title{
High reproducibility of patellar tendon vascularity assessed by colour Doppler ultrasonography: a reliable measurement tool for quantifying tendon pathology
}

\author{
J L Cook, R Ptazsnik, Z S Kiss, P Malliaras, M E Morris, J De Luca
}

Br J Sports Med 2005;39:700-703. doi: 10.1136/bjsm.2004.016410

See end of article for authors' affiliations

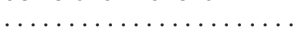

Correspondence to: Jill L Cook, La Trobe University, Bundoora, Victoria, Australia; i. cook@latrobe.edu.au

Accepted 22 February 2005

\begin{abstract}
Background: This study examined whether patellar tendon vascularity could be quantified accurately in the clinical setting using colour Doppler ultrasonography.

Methods: A sonographer and two radiologists visually estimated tendon vascularity in millimetres in 74 tendons during ultrasound (US) examination and from hard copy films. These estimates were then compared to the length of vessels measured from the digital image in millimetres and the correlation between them was determined. A subset of 16 tendons was used to compare the estimates of vascularity by two examiners at US examination.

Results: The estimation of vascular length at US examination correlated highly with the measured vascular length ( $r=0.92$; $95 \%$ confidence interval $(\mathrm{Cl}) 0.87$ to 0.94$)$, as did the length estimated from the films ( $r=0.94 ; 95 \% \mathrm{Cl} 0.9$ to 0.96$)$. The correlation between examiners was 0.84 (95\% $\mathrm{Cl} 0.51$ to 0.94 ) for the estimates made during US examination and $0.85(95 \% \mathrm{Cl} 0.59$ to 0.95$)$ for the vessel lengths measured from the digital images.

Conclusions: These excellent correlations indicate that tendon vascularity can be reliably estimated using colour Doppler ultrasonography and tendon vascularity could therefore be used by clinicians to rate clinical change. This method of quantifying tendon vascularity could also be used in research to investigate the effects of tendon treatments on vascularity.
\end{abstract}

$\mathrm{N}$ ormal tendons are not painful and have no visible blood vessels when examined with colour Doppler ultrasound (US). ${ }^{1}$ In contrast, pain has been associated with tendon vascularity in chronic Achilles tendinopathy, and sclerosis of these vessels has been shown to significantly decrease pain. ${ }^{2}$ Although tendon vascularity can be a feature of patellar tendinopathy, ${ }^{3-5}$ the relationship between tendon pain and vascularity is not completely clear in the patellar tendon and further investigation is warranted.

Currently, there are no reliable and valid measurement tools to quickly quantify vascularity in the clinical or research setting. Previous studies of tendon vascularity have not attempted to quantify the extent of the vascularity; tendons were simply classified as normal or abnormal. ${ }^{46}$ Tendons with florid vascularity or no vascularity are simple to classify. Clinically, however, a range of tendon vascularity is encountered, from small single vessels to complex and exuberant vascularity.

A method to quantify tendon vascularity is essential if the importance of vascularity in patients presenting with painful tendons is to be explored. Quantification of changes in vascularity over time in these patients may allow a better understanding of the role of vascularity in tendon morbidity. This may in turn allow improved clinical decision making when deciding on conservative or surgical management. Tendon research would also be assisted by a measurement system that reliably quantifies tendon vascularity, as it would allow further examination of the role of vascularity in tendon pain, pathology, and recovery.

A measure of tendon vascularity must be reliable and correctly estimate the amount of vascularity in a tendon when compared to a measure of known accuracy. The measure must also return comparable scores when used by more than one examiner. The primary aim of this study was to establish a reliable, clinically relevant, and time efficient method of estimating tendon vascularity. The secondary aim was to compare the clinical and measured vascular scores of two clinicians examining the same tendon.

\section{METHODS}

This study compared (i) an estimate of tendon vascularity at US examination, (ii) an estimate of tendon vascularity taken from the films of the same examination, and (iii) a direct measurement of the vascularity conducted on a computer (fig 1). The study also compared the estimates of vessel length at US examination by two examiners.

\section{Subjects}

The patellar tendons of 120 actively competing volleyball players (three grades from elite to domestic competition) were examined with real time US by an experienced musculoskeletal sonographer. Both patellar tendons for each player were imaged.

Subjects were part of a larger cohort that included those with and without tendon pathology and with and without knee symptoms. Subjects' knee pain have not been reported as this paper is intended to only report a measurement technique and not reflect on the clinical presentation of the subjects. Ethics approval was obtained from the La Trobe University Human Ethics Committee and all subjects provided informed consent.

\section{Ultrasound protocol}

The tendons were initially imaged on an US machine in both grey scale and colour Doppler with a $13.5 \mathrm{MHz}$ linear transducer (Siemens Acuson CV70, Siemens, Germany). Colour Doppler settings were standardised with a gain of

Abbreviations: $\mathrm{Cl}$, confidence interval; ICC, intraclass correlation coefficient; US, ultrasound 
Image

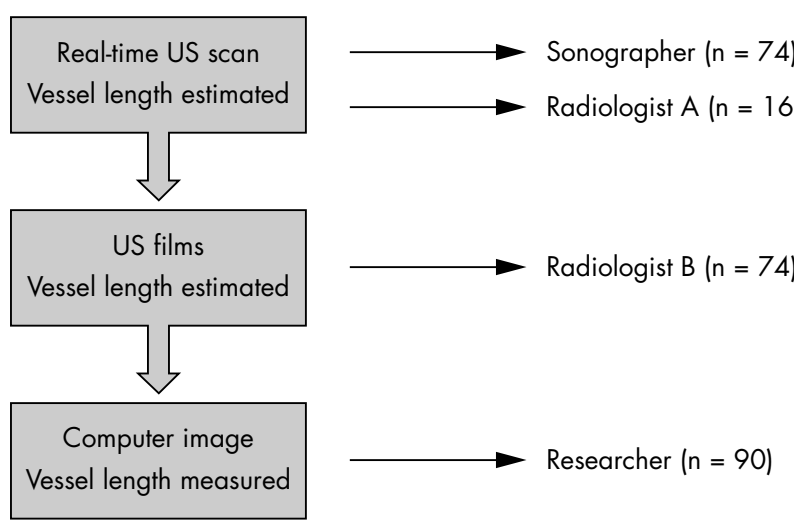

Figure 1 A flow diagram showing the images and personnel involved in this study.

$68 \mathrm{~dB}$, a sensitivity of $8 \mathrm{~cm} / \mathrm{s}$, and a pulse repetition frequency of $1250 \mathrm{~Hz}$. These settings were chosen as they represent the mid range of settings used to demonstrate tendon vascularity and can image vascularity without excessive artefact. This allowed for a direct comparison of the tendon vascularity between individuals.

Subjects were imaged in supine position with the knee fully extended; care was taken to ensure the quadriceps were fully relaxed. All tendons included in this study were hypoechogenic, a state which has been highly correlated with abnormal histopathology. ${ }^{7}$ All US examinations and estimations were conducted by the same investigator (sonographer, 20 years' experience), as were the US films (radiologist, 25 years' experience) and the measured score (researcher). The two investigators used to examine the same tendon were the sonographer and another radiologist with 35 years' experience. All images were then recorded to compact disc.

\section{Measurement protocol}

The protocol used to quantify tendon vascularity was the same for each of the three measures used (fig 2). Tendons were only measured from the sagittal plane image.

Each vessel visible in the tendon was quantified (estimated or measured) in millimetres. Vessels estimated or measured to be less than $1 \mathrm{~mm}$ in length were not included, while vessels that were not continuous but had breaks of less than $1 \mathrm{~mm}$ between ends were considered to be continuous. Vessels clearly in the fat pad or superficial to the tendon were not counted, while those whose location was more difficult to determine were considered to be tendon vessels. The lengths of all vessels were totalled to give a measure of tendon vascularity for each tendon.

To standardise the area measured, a $15 \mathrm{~mm}$ (AP) by $20 \mathrm{~mm}$ focus box was placed on the sagittal image with the greatest vascularity, centred over the proximal aspect of the tendon, and only vessels within this box were measured or estimated for all vascularity measures.

\section{Vessel length estimated from US imaging}

The vessel lengths visually estimated during real time US examination and from US films were done blind to the other estimates or measures. The examiners estimated the vessel length by placing one line of known length on the image (usually $5 \mathrm{~mm}$ ) and then, using this line as a guide, estimating the total length of the vessels. The vessel length estimated from the US films took account of magnification of the image.
For inter-tester reliability, the same procedure was then repeated by a radiologist and included US examination of the tendon and selection of the image for vessel length estimation; these procedures were completed blind to the sonographer's images or estimates.

\section{Measured vessel length}

The stored images were viewed in Photoshop (Adobe version 7) and measured blind to the imaging estimates. Using the software's measurement tool, the $15 \mathrm{~mm}$ box was used to determine the number of pixels for $1 \mathrm{~mm}$. Tortuous vessels (those that changed direction several times) were measured with a maximum of three lines and the score totalled to give the vessel an overall score (fig 2). All tendon vessels were measured in pixels, and the total added and converted to a length in millimetres. All images were measured twice by the researcher to establish the test-retest reliability of the measured score.

\section{Data analysis}

Data were entered into SPPS (Version 10) and mean and standard deviation of the vessel lengths calculated. The correlation of the vessel length estimated from the US examination and from the film with that measured from the stored images was determined using Pearson's r. The vessel length was measured twice on the computer and the two measures were compared with a two way mixed model intraclass correlation coefficient (ICC) for absolute agreement (test-retest). The vessel lengths estimated by the two examiners during US examination were compared with an ICC (two way random model) for absolute agreement (intertester).

\section{RESULTS}

Seventy four vascular tendons (39 right, 35 left) were included in the analysis. Sixteen of these tendons were examined by two examiners to determine the inter-tester reliability of the vascular measurements.

There was no significant difference between the mean of the estimates of vessel length at US examination, the estimates of the vessel length on the films, and the measured length using the computer (table 1). This suggests that each method of quantifying vessel length gave similar results.

This is supported by the excellent correlation between all three methods of quantifying vessel lengths (table 2); the correlation between all measurements was significant at $\mathrm{p}<0.01$.

The test-retest reliability of the measured vessel length (0.94; 95\% confidence interval (CI) 0.88 to 0.97) was excellent, as was the inter-tester reliability between the two examiners of the estimated vessel length at US examination $(0.84 ; 95 \%$ CI 0.51 to 0.94$)$ (fig 3 ). There were no significant differences between the estimates from US examination $(p=0.17)$ and measured lengths $(p=0.45)$ of the two examiners (table 3 ).

\section{DISCUSSION}

This study showed that estimating vessel length from US examination or films is a valid method for quantifying tendon vascularity. Therefore, these easy and time efficient measures can be used to quantify tendon vascularity in the clinical setting as quick and reliable quantification across a wide range of tendon vascularities is possible.

This study also demonstrates that two clinicians can image the same tendon and provide similar estimates of tendon vessel length. Despite differences in the appearance of the vessels in the actual images, this method returned comparable measures (fig 3 ). 
Table 1 Mean (SD) of the three sagittal plane scores

\begin{tabular}{lllr}
\hline Vessel length & $\mathbf{n}$ & $\begin{array}{l}\text { Range, } \\
\mathbf{m m}\end{array}$ & $\begin{array}{l}\text { Mean (SD), } \\
\mathbf{m m}\end{array}$ \\
\hline Measured on computer & 74 & $1-32.5$ & $9.9(7.3)$ \\
Estimated at US examination & 74 & $1-37$ & $13.4(9.0)$ \\
Estimated from US films & 72 & $1-30$ & $11.1(7.3)$ \\
\hline
\end{tabular}

Table 2 Correlation matrix for vessel length between the three forms of measurement (Pearson's r)

\begin{tabular}{llll}
\hline & $\begin{array}{l}\text { Measured } \\
\text { on } \\
\text { computer }\end{array}$ & $\begin{array}{l}\text { Estimated } \\
\text { at US } \\
\text { examination }\end{array}$ & $\begin{array}{l}\text { Estimated } \\
\text { from US } \\
\text { films }\end{array}$ \\
\hline $\begin{array}{l}\text { Measured on computer } \\
\begin{array}{l}\text { Estimated at US } \\
\text { examination }\end{array}\end{array}$ & $\begin{array}{l}0.92^{*} \\
\text { Estimated from US films }\end{array}$ & 1 & $0.93^{*}$ \\
\hline *Significant at $\mathrm{p}<0.01$. & & 1 \\
\hline
\end{tabular}

Table 3 Vessel length estimated from US examination and measures from computer images by two examiners

\begin{tabular}{|c|c|c|}
\hline & $\begin{array}{l}\text { Estimated from } \\
\text { US examination }\end{array}$ & $\begin{array}{l}\text { Measured } \\
\text { on computer }\end{array}$ \\
\hline Sonographer & $20.2(12.7)$ & $15.1(9.3)$ \\
\hline Radiologist & $17.0(10.3)$ & $16.5(10.3)$ \\
\hline
\end{tabular}

$\mathrm{n}=16$; values are in millimetres and are mean (SD).

This study fills a gap in imaging tendons and their vascularity, as early studies on tendon vascularity have not attempted to scale vascularity as "there have been no reports of a reliable system for grading vascularity". ${ }^{8}$ This scoring system will allow clinicians and researchers to further investigate treatments that may affect tendon vascularity and pain.

The sagittal plane was chosen as the primary scan plane for the clinical length because most tendon vessels lie in this plane. Axial images were taken and analysed, but provided no improvement on the reported measurement technique.

The clinical estimators of tendon vascularity in this study had limited experience and training in estimating vessel length and had not cross referenced their methods prior to this investigation. This suggests that this method can be learned and become clinically reliable in a short time with little training. This increases the clinical utility of the method as no intense training appears to be required to record reproducible tendon vascularity estimates.

\section{Limitations of this study}

Methods to account for vessel diameter were not included this measurement technique as they would have decreased the clinical utility. Further research on vascular diameter is required to clearly demonstrate its association with tendon pain and morbidity.

This study used standardised colour Doppler gain and colour. It is unclear if other colour Doppler settings would impact on the validity of this method as only vessel length is measured. However, it is likely that this measuring system would be resilient to changes in colour Doppler settings. It is important to note that repeat estimates and measurements of tendon vascularity over time must be carried out with the same colour Doppler settings.

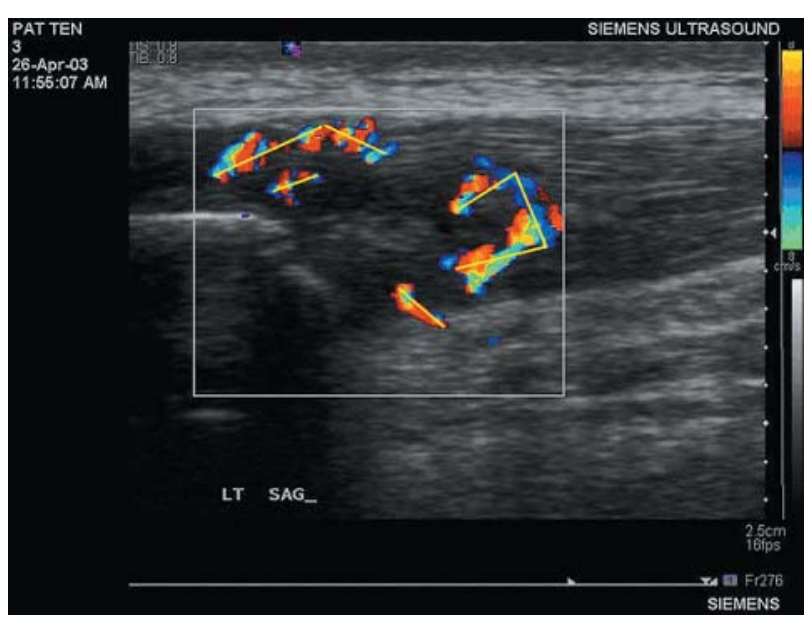

Figure 2 The measuring technique used when estimating or measuring tendon vessel length. When measuring on the computer, a maximum of three lines was used to quantify a single vessel. The total lengths of line were then summed to give a measure of tendon vascularity.

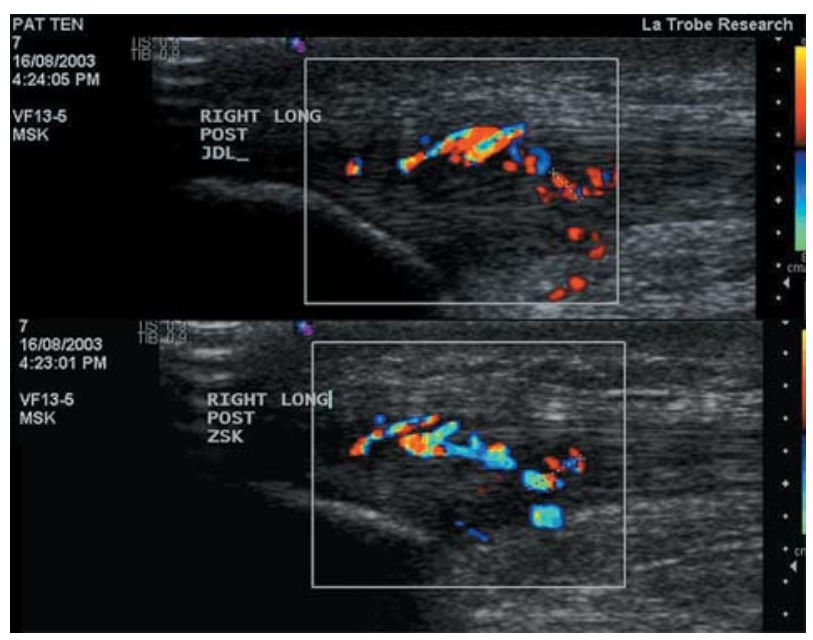

Figure 3 The images of a single tendon selected by the two examiners, clinically estimated, and then measured. The estimated vessel lengths at US examination for these images were 19 and $22 \mathrm{~mm}$ and the measured lengths on computer were 20 and $22 \mathrm{~mm}$.

These tendon vascularity measures have been examined as regards the patellar tendon only, and their applicability to other tendons must be determined. However, the simplicity and utility of this measurement indicate that it may be equally applicable to other tendons.

\section{CONCLUSIONS}

Estimating tendon vascularity at US examination and from US films is reliable and valid and has utility for both the clinical setting and research on tendon vascularity. Further investigation of tendon vascularity, pain, and morbidity may be more easily quantified now that a reliable and valid measurement tool is available for this purpose.

\section{Authors' affiliations}

J L Cook, P Malliaras, M E Morris, La Trobe University, Bundoora, Victoria, Australia

R Ptazsnik, Monash University, Victoria, Australia

Z S Kiss, Medical Imaging Australia, Melbourne, Australia

J De Luca, La Trobe University Medical Centre, Medical Imaging,

Victoria, Australia 


\section{What is already known on this topic}

Previously, there have been no reliable and valid measurement tools to quickly quantify vascularity in the clinical or research setting.

\section{What this study adds}

Thus study reports that tendon vascularity can be reliably estimated using colour Doppler ultrasonography.

The authors value the support of Seimens $\mathrm{P} / \mathrm{L}$ who provided the ultrasound machine for this study free of charge

Competing interests: none declared

\section{REFERENCES}

1 Ohberg L, Lorentzon R, Alfredson H. Neovascularisation in Achilles tendons with painful tendinosis but not in normal tendons: an ultrasonographic investigation. Knee Surgery Sports Traumatol Arthrosc 2001:9:233-8.

2 Ohberg L, Alfredson $\mathrm{H}$. Ultrasound guided sclerosis of neovessels in painful chronic Achilles tendinosis: pilot study of a new treatment. Br J Sports Med 2002;36: 173-7.

3 Weinberg EP, Adams MJ, Hollenberg GM. Color Doppler sonography of patellar tendinosis. Am J Roentgenol AJR 1998;171:743-4.

4 Terslev L, Qvistgaard E, Torp-Pedersen S, et al. Ultrasound and power Doppler findings in jumper's knee - preliminary findings. Eur J Ultrasound 2001;13:183-9.

5 Cook JL, Malliaras P, Luca JD, et al. Neovascularisation and pain in abnormal patellar tendons of active jumping athletes. Clin J Sports Med 2004; 14(5):296-9.

6 Khan KM, Forster BB, Robinson J, et al. Are ultrasound and magnetic resonance imaging of value in assessment of Achilles tendon disorders? A two year prospective study. Br J Sports Med 2003;37(2): 149-54.

7 Khan KM, Bonar F, Desmond PM, et al. Patellar tendinosis (jumper's knee): findings at histopathologic examination, US and MR imaging. Radiology 1996:200:821-7.

8 Zanetti M, Metzdorf A, Kundert H-P, et al. Achilles tendons: clinical relevance of neovascularization diagnosed with power Doppler US. Radiology 2003;227:556-60.

\section{bmjupdates+}

bmjupdates+ is a unique and free alerting service, designed to keep you up to date with the medical literature that is truly important to your practice.

bmjupdates+ will alert you to important new research and will provide you with the best new evidence concerning important advances in health care, tailored to your medical interests and time demands.

\section{Where does the information come from?}

bmjupdates+applies an expert critical appraisal filter to over 100 top medical journals A panel of over 2000 physicians find the few 'must read' studies for each area of clinical interest

Sign up to receive your tailored email alerts, searching access and more...

www.bmjupdates.com 